\title{
Impact of COVID-19 on rheumatology practice in the UK-a pan-regional rheumatology survey
}

\author{
Arvind Nune $^{1}$ (D) $\cdot$ Karthikeyan P. lyengar $^{1}$ (D) $\cdot{\text { Ashar } \text { Ahmed }^{1} \cdot \text { Syed Bilgrami }}^{2} \cdot$ Hem Raj Sapkota $^{3}(\mathbb{C}$
}

Received: 12 December 2020 / Revised: 17 January 2021 / Accepted: 18 January 2021 / Published online: 25 January 2021

(C) International League of Associations for Rheumatology (ILAR) 2021

\begin{abstract}
The COVID-19 pandemic has disrupted healthcare services and rheumatology staff were redeployed to the frontline. The purpose of this survey was to evaluate the impact of the COVID-19 pandemic on the provision of rheumatology services as viewed by rheumatologists in the UK. Survey monkey questionnaire weblink was sent to 804 clinicians including consultant rheumatologists, speciality trainees, nurse specialists, and allied health professionals in 4 regions of the UK to evaluate personal effects of COVID-19 and redeployment, impact on current out-patient clinic activity, immunosuppressive drug use, and future rheumatology care. Response rate was $21 \%$. One-fifth of the responders reported that their rheumatology departments were functioning less than 50\% capacity during the pandemic. Two-third of responders felt anxious about the ill-effects of COVID-19 on their health and well-being, and one-third of them were redeployed. During the peak of the pandemic, $75 \%$ of clinicians stopped intravenous biologics. Although access to video consultation was available for up to three-fourths of the clinicians, the majority (90\%) used this modality in less than 1 in 4 consultations. This survey highlights rheumatologists' perception in the delivery of future care and anxiety they faced. As demonstrated by this survey, the National Institute for Health and Care Excellence (NICE) guidance did not influence clinician decision making in some aspects of patient care. Underutilization of tele-rheumatology in this survey should be considered whilst planning the restoration of rheumatology services in the post-COVID era.
\end{abstract}

Key Points

- COVID-19 has generated significant concerns among rheumatology community about their mental well-being.

- In over 50\% of cases, rheumatologists stopped IV biologic drugs as per patients' wishes during the first wave of the pandemic.

- Tele-rheumatology has been used more widely during the pandemic, but the extent of its use in the post-COVID era is less clear. Evolving evidence will determine its future wider use.

Keywords Biologics $\cdot$ COVID-19 $\cdot$ Pandemic $\cdot$ Surveys and questionnaires $\cdot$ Tele-rheumatology

Arvind Nune

arvind.nune@nhs.net

Karthikeyan P. Iyengar

Kiyengar@nhs.net

Ashar Ahmed

Ashar.ahmed1@nhs.net

Hem Raj Sapkota

hem.sapkota@nhs.net

1 Consultant Rheumatologist, Southport and Ormskirk NHS Trust, Southport PR8 6PN, UK

2 Consultant Rheumatologist, Royal Lancaster Infirmary, Lancaster LA1 4RP, UK

3 Consultant Rheumatologist, The Royal Wolverhampton NHS Trust, Wolverhampton WV10 0QP, UK

\section{Introduction}

As the new coronavirus SARS-CoV-2 (COVID-19) pandemic reached the United Kingdom (UK), its effect on the National Health Service (NHS) and restrictions imposed to curtail the spread of the viral disease have been profound [1]. To reduce the risk of person-to-person transmission of the virus, Centers for Disease Control and Prevention (CDC) and Public Health England (PHE) introduced guidelines and measures such as 'social distancing' and use of personal protective equipment in health care environments [2, 3]. National Institute for Health and Care Excellence (NICE) produced rapid COVID-19 guidelines for managing patients with rheumatological autoimmune inflammatory and metabolic bone disorders during the COVID-19 global pandemic [4]. To deliver safe and 
effective care to patients, a balance must be achieved between protecting patients and staff from viral transmission whilst providing a continuity of care. Rheumatology services across the UK had to be reconfigured and reorganised to rise to these challenges [5]. This survey evaluates the impact of the pandemic on rheumatology service provision as perceived by rheumatologists in the UK and various strategies adopted to provide effective clinical care.

\section{Material and Methods}

\section{Study design}

This cross-sectional study used an online questionnaire consisting of 37 questions (supplemental material). The questionnaire was developed using survey monkey and disseminated to 804 clinicians in rheumatology in four regions of the UK (Manchester/Northwest, Liverpool/Mersey, Midlands and Wales) through electronic mail. The survey was aimed at rheumatology consultants, speciality trainees, associate specialist/staff grade doctors, nurse specialists, and allied healthcare professionals. The survey allowed responses for 4 weeks from 18 August 2020.

\section{Data collection}

The responses submitted were checked for duplication, pooled, analysed, and summarised.

\section{Evaluation points}

The focus of the survey was on the following points:

(a) Personal effects of COVID-19 and redeployment of rheumatology health care professionals

(b) Impact on current out-patient clinic activity

(c) Impact on immunosuppressive drug use

(d) Impact on future rheumatology care

\section{Results}

The survey was completed by 172 out of 804 rheumatology health care professionals (HCP) with a $21 \%$ response rate. All responses received were used in the analysis. $68 \%$ of the responders were female. Nearly half of the total responders were consultant grade (Table 1).
Table 1 Characteristics of the participants $(n=172)$

\begin{tabular}{|c|c|c|}
\hline Age (years) & $N(\%)$ & \\
\hline$<40$ & 38 & $(22.5)$ \\
\hline $40-50$ & 60 & $(35)$ \\
\hline $50-60$ & 56 & (33) \\
\hline $60-70$ & 15 & (9) \\
\hline$>70$ & 01 & $(0.5)$ \\
\hline Skipped & 02 & \\
\hline \multicolumn{3}{|l|}{ Gender } \\
\hline Male & 54 & (32) \\
\hline Female & 116 & $(68)$ \\
\hline Skipped & 02 & \\
\hline \multicolumn{3}{|l|}{ Professional grade } \\
\hline Consultant & 83 & $(49)$ \\
\hline Associate specialist/specialty doctor & 09 & $(5.3)$ \\
\hline Specialty trainee & 20 & $(11.8)$ \\
\hline Nurse specialist & 42 & $(24.8)$ \\
\hline Allied health professionals & 15 & $(8.8)$ \\
\hline Skipped & 03 & \\
\hline \multicolumn{3}{|l|}{ Ethnicity } \\
\hline White & 117 & $(68.4)$ \\
\hline Asian/Asian British & 40 & $(23.39)$ \\
\hline Black/Black British & 05 & $(2.92)$ \\
\hline Mixed & 03 & $(1.75)$ \\
\hline Other & 05 & $(2.92)$ \\
\hline Skipped & 01 & \\
\hline \multicolumn{3}{|l|}{ Area of work } \\
\hline Manchester/North West & 55 & $(32.74)$ \\
\hline Liverpool/Merseyside & 45 & $(26.79$ \\
\hline Midlands & 52 & $(30.95)$ \\
\hline Wales & 16 & $(9.53)$ \\
\hline Skipped & 04 & \\
\hline
\end{tabular}

\section{Personal effects of COVID-19 and redeployment of rheumatology health care professionals}

57 (37\%) clinicians were redeployed to the frontline, and most of them helped cover the COVID-19 wards. Although there was a negative press about personal protective equipment (PPE), most $(70 \%)$ responders reported that they were satisfied with PPE's level provided based on patient care during the peak of the pandemic. Over half of the rheumatologists reported that they would consider themselves at high risk of contracting coronavirus SARS-CoV-2. $60 \%$ of clinicians felt anxious about the ill-effects of coronavirus on their health and well-being, but two-thirds of them were not anxious or concerned about face-to-face consultations. 


\section{Impact on current out-patient clinic activity}

One-fifth of the responders reported that their rheumatology departments were functioning less than $50 \%$ capacity during the pandemic, with $10 \%$ of the staff were either shielding or self-isolating due to health reasons.

\section{Tele-rheumatology}

Up to $80 \%$ of clinicians discontinued face-to-face appointments but continued telephone consultations to minimise viral transmission during the peak of the pandemic.

$50 \%$ of clinicians reported using the telephone consultations for over three-fourths of their follow-up patients; however, just over $10 \%$ of clinicians utilised the telephone for new consultations for the same number of patients. In contrast, video consultations were massively underutilised for both new and follow-up patients, despite widely available to $70 \%$ of participants. $90 \%$ of clinicians reported using video consultations for both new and follow-up patients in less than 1 in 4 cases (Fig. 1).

\section{Impact on immunosuppressive drug use}

114 (77\%) rheumatologists stopped intravenous (IV) biologics during the peak of the pandemic. Of these, in 50\% cases, patients requested this, and in $27 \%$ of cases, clinicians decided to stop the intravenous biologic treatment. $60 \%$ of clinicians converted IV biologics to subcutaneous (SC) forms during the pandemic.

\section{Impact on future rheumatology care}

Over $75 \%$ of clinicians predicted that only a quarter of their future new patient appointments would be telephone or video consultations (Fig. 2). 50\% of clinicians were using telephone consultations for their follow-up patients in three-fourth of cases during the peak of the pandemic, but less than $10 \%$ of them would expect to do so in future.

\section{Discussion}

This survey provides an insight into the concerns of rheumatologists' health and well-being and the challenges they faced in delivering rheumatology care during the pandemic and prospects for future rheumatology care.

Although the majority of clinicians in our study reported that they had moderate to severe anxiety about the implications of coronavirus on their health (graded anxiety over 3 with 5 being severely anxious), only a small proportion $(1.3 \%)$ sought medical help. Clinicians appear to have been concerned about general ill-effects of the pandemic on their health and well-being and the risk of contracting the virus whilst being redeployed to the medical wards. Also, up to half of the clinicians in our study were over 50 , just over $25 \%$ were BAME (Black, Asian, and Minority Ethnic) group and majority were men. These have noted to be independent risk factors for contracting coronavirus SARS-Cov-2 in various studies, and this could have been the reason for concern in our responders. However, the response suggests that many clinicians had no anxieties in engaging with face-to-face consultations.

Initial studies from China [6] established that HCP suffered psychological consequences such as anxiety and depression when directly engaged in managing the patients with COVID19. Redeployment, staff shielding, and isolation seemed to be two main reasons for short staffing and reduced clinical capacity in our study, which must have contributed to increased workload and anxiety. Many studies so far have evaluated
Fig. 1 Clinicians' current consultation types

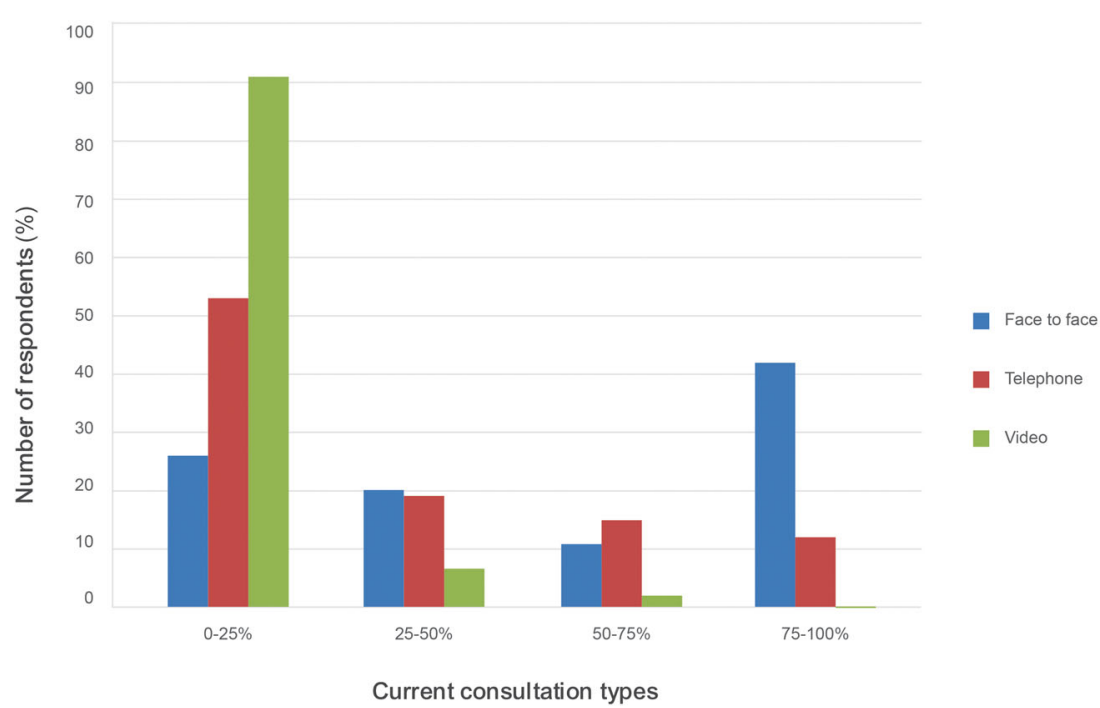


Fig. 2 Clinicians' future consultation types

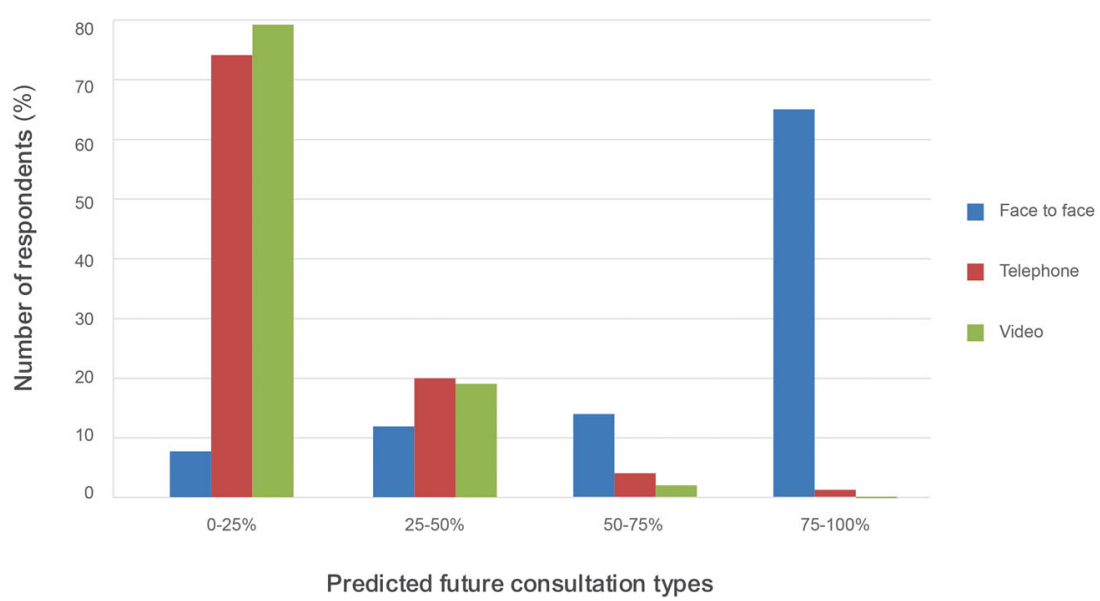

how the COVID-19 pandemic has adversely affected clinicians' health and well-being. Vindegaard et al., in their systematic review, reported that HCP in many observational studies suffered from mental health ailments such as anxiety and depression during the COVID-19 pandemic [7]. Although we did not explore why clinicians did not seek help for mental health issues, recent studies have highlighted that HCP generally avoid seeking help for mental health ailments due to fear of stigma and discrimination [8].

Telemedicine has revolutionised the way we deliver outpatient care [9]. Tele-rheumatology had been sporadically used in a small proportion of rheumatology patients in the pre-COVID era [10]. However, available data suggest that clinicians have adapted to this new way of consulting their patients and telemedicine is now widely used in rheumatology practice in many parts of the world $[11,12]$. Majority of clinicians in our study reported that they had access to telephone and video consultations; however, it appears that they were underutilised particularly for new patient consultations. This pattern was also observed in veteran affairs study from the USA [12]. Nevertheless, telephone consultations were the preferred choice for follow-up consultations in our study. There seems to be a difference of opinion in what patients and rheumatologists want to choose telemedicine or face-to-face consultations for rheumatology conditions. In an Italian study, over $60 \%$ of patients with connective tissue diseases (CTD) preferred teleconsultations over face-to-face appointments [11]. On the contrary, over $50 \%$ of clinicians in the veteran affairs study believed that their preferred mode of consultation for CTD patients was face-to-face consultations. Although further research is required to establish the safe delivery of tele-rheumatology in various rheumatology conditions such as rheumatoid arthritis (RA) and CTD, the recent promotion of video consultations by NICE [13] might help in the evaluation of rheumatology patients, for example the nature of skin rashes in CTD or joint swellings in RA.

Contrary to the studies [14] forecasting the rise of telerheumatology, the majority of clinicians in our survey predicted that teleconsultations would be perhaps the least preferred mode of future consultations. Exact reasons for underuse of telemedicine in future are unclear, but this could be due to several factors including elderly patients who may not have good hearing, lack of internet access, poor quality of the image in video consultations, and network issues. Lack of access to the physical examination could have been another potential reason for the reluctance of tele-rheumatology in future, particularly when consulting new patients. As discussed above, most clinicians were not anxious or concerned about the adverse effects of coronavirus during faceto-face consultations which could have influenced their views on the future use of tele-rheumatology. As promoted by NHS England, we feel that a teleconsultation is a useful tool and this should be considered for new patient consultations either to triage or for an initial consultation where appropriate to minimise viral transmission [15]. It is acknowledged that telemedicine technology should be applied in appropriate settings and situations. However, suitable training, enhanced documentation, and applying information governance will go a long way in avoiding pitfalls associated with remote consultations [16].

To our knowledge, our study is the first national survey evaluating the views of clinicians about their choice of rheumatology treatment use during the pandemic and postpandemic rheumatology practice. Most clinicians' decision to stop IV biologic drugs during the pandemic's peak is understandable due to the requirement for these patients to attend hospitals for their infusions. In most cases, patients were given the option to change their treatment to SC form to allow selfadministration. This was probably to protect vulnerable patients from hospital exposure, simultaneously reducing patient foot-fall in hospitals allowing for more effective social distancing measures. Early in the pandemic, there was lack of evidence whether immunosuppressive drugs could be continued safely although NICE guidelines advised continuing patients on their biologic therapy. The risk of infections was thought to be slightly raised when patients with chronic inflammatory arthritis are treated with biologic anti-rheumatic 
drugs, particularly in elderly patients with co-morbidities [17]. The rationale to continue biologic drugs is mainly based on expert opinion and their clinical experience with the notion that the continuation of immunosuppressive drugs would minimise the disease flares and subsequent hospital admissions during the pandemic $[5,18]$. Although we did not specifically ask whether clinicians continued other forms of biologics, over $60 \%$ of clinicians favoured switching IV to SC biologics which is in line with NICE recommendation [4]; therefore, we can assume that most clinicians continued SC or oral forms of biologics for their patients. Majority of rheumatologists $(N=80$, $58 \%$ ) indicated that their recommendation for patients' rheumatic drug choice would change due to the risk of COVID-19 infection. $64 \%$ of clinicians prefer to use either SC biologics or oral Janus kinase inhibitors/phosphodiesterase inhibitors (JAK/ PDE4) over IV biologics to minimise the hospital visits. Therefore, we envisage that rheumatology care pathways will adapt according to the evolving evidence, patients' requirements, and ongoing risk of COVID-19 infection. One in two clinicians preferred to use oral steroids over IM or IV forms, although longterm corticosteroids are thought to adversely affect rheumatology patients' outcome during the pandemic [19].

\section{Limitations of the study}

Our survey has some inherent limitations due to its nature. The response rate was low, and therefore, the data may not be generalisable to a wider community. As with any survey, responders may have misinterpreted and skipped some of the questions with inaccurate responses.

Speciality trainees $(20 ; 11 \%)$ and clinicians from black ethnicity were under-represented. The majority of responders were rheumatology consultants which may slightly reduce the applicability of the data to other team members. We did not have a breakdown of the four regional rheumatology society subscribers where the survey weblink was sent. Therefore, we are unable to compare responses between responders and nonresponders. Although consultant rheumatologists usually lead the clinical management decisions, poor representation of the trainees and low response rate might have skewed the results, particularly for future rheumatology care. Despite this, we believe results will help derive meaningful conclusions about the challenges faced in the current practice and future directions. Even though patients might have equally concerned about continuing of synthetic DMRDs during the first peak of the pandemic, we did not evaluate this in our survey.

\section{Conclusion}

Amid current pandemic, one of the largest rheumatology clinician surveys of this kind reflects the concerns among rheumatology community regarding staffing, health and well- being, and current and future rheumatology practice. As demonstrated by this survey, NICE guidance did not influence clinician decision making in some aspects of patient care. Respondents in our survey did not favour tele-rheumatology for both new and follow-up patients. Although our study demonstrates a variation of immunosuppressive drug use, emerging new evidence will guide rheumatology practice and therapy choice.

Supplementary Information The online version contains supplementary material available at https://doi.org/10.1007/s10067-021-05601-1.

Author contributions AN and KI involved in conceptualization, literature search, manuscript writing, and editing. AA and SB involved in literature search, manuscript writing, and references. HS supervised the overall submission and edited and approved the final draft. All authors read and agreed the final draft submitted.

\section{Compliance with ethical standards}

Disclosures None.

Ethics approval and consent to participate Although discussed with research and development team, ethics approval was not required for this study.

\section{References}

1. Public Health England. UK (2020). Accessed on 29 November 2020. Available from: https://www.gov.uk/coronavirus

2. Centers for Disease Control and Prevention USA. (2020). Coronavirus disease 2019 (COVID-19) prevention and treatment. Accessed on 29 November 2020. Available from: https://www.cdc. gov/coronavirus/2019-ncov/index.html

3. Public Health England. UK. (2020). Coronavirus (COVID-19): personal protective equipment (PPE) hub. Accessed on 29 November 2020. Available from: https://www.gov.uk/ government/collections/coronavirus-covid-19-personal-protectiveequipment-ppe

4. National Institute for Health and Care Excellence (NICE 2020) COVID-19 rapid guideline: rheumatological autoimmune, inflammatory and metabolic bone disorders (NG167) Available from https://www.nice.org.uk/guidance/ng167

5. Nune A, Iyengar K, Ahmed A, Sapkota H (2020) Challenges in delivering rheumatology care during COVID-19 pandemic. Clin Rheumatology 39:2817-2821

6. Lai J, Ma S, Wang Y, Cai Z, Hu J et al (2020) Factors associated with mental health outcomes among health care workers exposed to coronavirus disease 2019. JAMA Netw Open 3:e203976

7. Vindegaard N, Benros ME (2020) COVID-19 pandemic and mental health consequences: systematic review of the current evidence. Brain Behav Immun 89:531-542

8. Taylor WD, Blackford JU (2020) Mental Health treatment for front-line clinicians during and after the coronavirus disease 2019 (COVID-19). Pandemic: A Plea to the Medical Community. Ann Intern Med 173:574-575

9. Maese JR, Seminara D, Shah Z, Szerszen A (2020) Perspective: what a difference a disaster makes: the telehealth revolution in the age of COVID-19 pandemic. Am J Med Qual 35:429-431 
10. McDougall JA, Ferucci ED, Glover J, Fraenkel L (2017) Telerheumatology: a systematic review. Arthritis care \& research 69:1546-1557

11. Cavagna L, Zanframundo G, Codullo V, Pisu MG, Caporali R et al. (2020). Telemedicine in rheumatology: a reliable approach beyond the pandemic. Rheumatology (Oxford). 7: keaa554. DOI: https:// doi.org/10.1093/rheumatology/keaa554

12. Singh JA, Richards JS, Chang E, Joseph A, Ng B. (2020). Management of rheumatic diseases during the COVID-19 pandemic: a National Veterans Affairs Survey of rheumatologists. Arthritis Care Res (Hoboken). 15:https://doi.org/10.1002/acr.24487 doi: https://doi.org/10.1002/acr.24487

13. NHS England and NHS Improvement (2020). Attend anywhere. Available from: https://england.nhs.attendanywhere.com/ resourcecentre/Content/Public_Topics/Discover.htm. [Accessed 29/11/2020]

14. Gkrouzman E, Wu DD, Jethwa H, Abraham S (2020) Telemedicine in rheumatology at the advent of the COVID-19 pandemic. HSS J $6: 1-4$

15. Remote consultations NHS England and NHS improvement coronavirus. (2020). specialty guides for patient management. https:// www.england.nhs.uk/coronavirus/publication/specialty.guides/ Available
16. Iyengar K, Jain VK, Vaishya R (2000) Pitfalls in telemedicine consultations in the era of COVID 19 and how to avoid them. Diabetes Metab Syndr 14:797-799. https://doi.org/10.1016/j.dsx.2020.06.007

17. Quartuccio L, Zabotti A, Del Zotto S, Zanier L, De Vita S et al (2018) Risk of serious infection among patients receiving biologics for chronic inflammatory diseases: usefulness of administrative data. J Adv Res 15:87-93. https://doi.org/10.1016/j.jare.2018.09.003

18. Favalli EG, Ingegnoli F, De Lucia O, Cincinelli G, Cimaz R et al (2020) COVID-19 infection and rheumatoid arthritis: faraway, so close! Autoimmun Rev 19:102523

19. Gianfrancesco MA, Hyrich KL, Gossec L, Strangfeld A, Carmona L, Mateus EF, Sufka P, Grainger R, Wallace Z, Bhana S, Sirotich E, Liew J, Hausmann JS, Costello W, Robinson P, Machado PM, Yazdany J, COVID-19 Global Rheumatology Alliance Steering Committee (2020) COVID-19 global rheumatology alliance steering committee. Rheumatic disease and COVID-19: initial data from the COVID-19 Global Rheumatology Alliance provider registries. Lancet Rheumatol 2:e250-e253

Publisher's note Springer Nature remains neutral with regard to jurisdictional claims in published maps and institutional affiliations. 